\title{
Kriterien zweiter Ordnung für lokal beste Approximationen
}

\author{
R. Hettich
}

Eingegangen am 20. Mai 1974

\begin{abstract}
Summary. Consider the problem of approximating (in the Chebyshev-norm) a real-valued function $f(x)$ on a compact subset $X$ of $\mathbb{R}^{m}, m \geqq 1$, by an element of a set of functions $a(p, x), p \in P, P \leq \mathbb{R}^{n}$ an open set. Both necessary and sufficient conditions of the second order for an $a\left(p^{0}, x\right)$ to be a locally best approximation are derived. Apart from conditions on the differentiability of $f$ and $a$, on $X$, and on the error function $f(x)-a\left(p^{0}, x\right)$ we impose no restrictions on the problem. This makes the results applicable to a broad class of problems.
\end{abstract}

\section{Einleitung}

In [5] und [1] wurden für die Tschebyscheff-Approximation Kriterien zweiter Ordnung für lokal beste Approximationen aufgestellt. Hierzu wurden solche Kriterien erst allgemein für nichtlineare Optimierungsaufgaben hergeleitet und dann auf das der Tschebyscheff-Approximation entsprechende Optimierungsproblem spezialisiert. Dabei zeigte sich, daß ein Teil der im allgemeinen Fall benötigten Voraussetzungen (sog. ",constraint qualifications") stets erfüllt ist. Insbesondere wurde in [1] gezeigt, da $\beta$ man diese recht unhandlichen Voraussetzungen vollständig ersetzen kann durch Bedingungen an die zugehörige Fehlerfunktion, die im konkreten Fall relativ einfach zu prüfen sind.

In der vorliegenden Arbeit fordern wir derartige Eigenschaften der Fehlerfunktion von vornherein und leiten die Kriterien direkt ab, d.h. ohne den Umweg über Optimierungsprobleme. Hierbei lassen wir weit allgemeinere (insbesondere mehrdimensionale) Approximationsbereiche als in [1] zu, wo nur die Approximation in reellen Intervallen betrachtet wurde.

Neben dem viel breiteren Anwendungsbereich haben die hier abgeleiteten Kriterien gegenüber dem Kriterium von Kolmogoroff noch folgenden wichtigen Vorteil: während dessen Nachprüfung eine Aussage über die gesamte Klasse der zur Approximation zugelassenen Funktionen erfordert (vgl. etwa [3]), werden hier nur Funktionswerte und Ableitungen der in Frage stehenden Funktion in den Extremalstellen der Fehlerfunktion benötigt (vgl. Satz 2 und 3). Allerdings erlaubt das Kriterium von Kolmogoroff Aussagen über global beste Approximationen.

\section{Bezeichnungen}

Der Ubersichtlichkeit halber wollen wir hier die wichtigsten der im folgenden gebrauchten Bezeichnungen zusammenstellen. Es bezeichnet

$x \quad$ einen Punkt des $\mathbb{R}^{m}$, meist $x \in B$

$x^{i} \quad$ die Punkte von $E \leq B$

$x^{j}(t) \quad$ Wege in $B, t \in \mathbb{R}, x^{j}(0)=x^{i} \in E$

30 Numer. Math., Bd. 22 
$\mu_{j}, \lambda_{j} \quad$ die ersten bzw. zweiten Ableitungen der $x^{j}(t)$ in $t=0$

$p, p^{0} \quad$ Punkte des Parameterbereichs $P \subseteq \mathbb{R}^{n}$

$p(t) \quad$ einen Weg in $P$

$\xi, \eta \quad$ die erste bzw. zweite Ableitung von $p(t)$ in $t=0$

$f(x) \quad$ die zu approximierende Funktion

$a(p, x)$ die Funktion, mit der approximiert wird

$e(p, x)$ die Fehlerfunktion; $e(p, x):=|f(x)-a(p, x)|$

$g^{i}(x) \quad$ Funktionen, die $B$ definieren, $i \in I$

$B$ den Bereich, in dem approximiert wird; $B \subset \mathbb{R}^{m}$

$P \quad$ den Parameterbereich; $P \leq \mathbb{R}^{n}$

$E$ die Menge der Punkte von $B$, in denen $e(p, x)$ maximal wird

$I, I(x)$ endliche Indexmengen, $I(x) \leq I$.

Ableitungen bezeichnen wir folgendermaßen:

- ein hochgestellter Punkt bedeutet die Ableitung nach $t$, z.B.

$$
\dot{x}^{i}(t)=\frac{d}{d t} x^{i}(t)
$$

- ein unterer Index $x$ bzw. $p$ bezeichnet den Gradienten bez. $x$ bzw. $p$ :

z.B. $g_{x}^{i}(x)=\left(\frac{\partial g^{i}(x)}{\partial x_{1}}, \ldots, \frac{\partial g^{i}(x)}{\partial x_{m}}\right)^{T}$ oder $e_{p}(p, x)=\left(\frac{\partial e(p, x)}{\partial p_{1}}, \ldots, \frac{\partial e(p, x)}{\partial p_{n}}\right)^{T}$

(ein hochgestelltes $T$ bedeutet den Übergang zur transponierten Matrix)

- ein unterer Index $x x, x p$ oder $p p$ bezeichnet die Matrizen der zweiten Ableitungen, z.B.

oder

$$
e_{x p}(p, x)=\left(\frac{\partial^{2} e(p, x)}{\partial x_{j} \partial p_{i}}\right), \quad j=1, \ldots, m ; i=1, \ldots, n(m \times n \text {-Matrix })
$$

$$
g_{x x}^{i}(x)=\left(\frac{\partial^{2} g^{i}(x)}{\partial x_{j} \partial x_{k}}\right), \quad j, k=1, \ldots, m(m \times m \text {-Matrix }) .
$$

Schließlich wollen wir vereinbaren, daß wir die Berechnung eines Funktionswertes für die Argumente $p=p^{0}, x=x^{i}$ durch einen hochgestellten Index $j$ und Weglassen der Argumente andeuten:

$$
\text { z.B. } e_{x p}^{j}=e_{x p}\left(p^{0}, x^{i}\right) \text { oder } g^{i, i}=g^{i}\left(x^{j}\right) .
$$

\section{Problemstellung}

Wir betrachten folgendes Approximationsproblem: $f(x)$ und $a(p, x)$ seien zweimal stetig differenzierbare, reellwertige Funktionen in $B C \mathbb{R}^{m}$ resp. $P \times B \subset \mathbb{R}^{n} \times \mathbb{R}^{m}$, wo $B$ kompakt und $P$ offen sei. Dann ist ein $p^{0} \in P$ zu bestimmen, so daB

$$
\left\|f-a\left(p^{0}, \cdot\right)\right\| \leqq\|f-a(p, \cdot)\|
$$

für alle $p$ im Durchschnitt einer Umgebung von $p^{0}$ mit $P$. Ein solches $p^{0}$ nennen wir eine lokal beste Approximation. Hierbei ist $\|\cdot\|$ die Maximumnorm: $\|g\|=$ $\max _{x \in B}|g(x)|$, für jede auf $B$ stetige Funktion $g$. 
Über $B$ machen wir folgende Voraussetzung:

(I). Es ist $B=\left\{x \in \mathbb{R}^{m} \mid g^{i}(x) \leqq 0, i \in I\right\}$ mit einer endlichen Indexmenge $I$. Die $g^{i}(x)$ seien im $\mathbb{R}^{m}$ zweimal stetig differenzierbare, reellwertige Funktionen. Jedem $x \in B$ ordnen wir die Indexmenge $I(x):=\left\{i \in I \mid g^{i}(x)=0\right\}$ zu. Für jedes $x \in B$ seien dann die Vektoren $g_{x}^{i}(x), i \in I(x)$, linear unabhängig.

Durch Forderung (I) werden im $\mathbb{R}^{2}$ etwa Bereiche mit nach innen oder nach außen gerichteten Spitzen, im $\mathbb{R}^{3}$ z.B. solche mit Oktaederecken, ausgeschlossen. Am Schlu $ß$ werden wir noch kurz darauf eingehen, wie man allgemeinere Bereiche in die Theorie einbeziehen kann.

Im folgenden betrachten wir ein festes $p^{0} \in P$. Die Frage ist, wie man entscheiden kann, ob $a\left(p^{0}, x\right)$ eine lokal beste Approximation ist. $Z \mathbf{u} p^{0}$ definieren wir die Fehlerfunktion

$$
e\left(p^{0}, x\right)=\left|f(x)-a\left(p^{0}, x\right)\right|,
$$

die in $B \sim\left\{x \mid e\left(p^{0}, x\right)=0\right\}$ zweimal stetig differenzierbar ist. Uber $e\left(p^{0}, x\right)$ machen wir folgende Voraussetzung, deren Bedeutung durch Hilfssatz 1 (unten) deutlich wird:

(II). Es sei $E=\left\{x \in B \mid e\left(p^{0}, x\right)=\left\|e\left(p^{0}, \cdot\right)\right\|\right\}$ und $\left\|e\left(p^{0}, \cdot\right)\right\|>0$. Dann gelte: zu jedem $x^{j} \in E$ gibt es $w_{i}^{j}>0, i \in I\left(x^{j}\right)$, mit

(i) $e_{x}^{i}-\sum_{i \in I\left(x^{i}\right)} w_{i}^{j} g_{x}^{i, j}=0$.

(ii) Auf dem Teilraum $T_{j}=\left\{\mu \in \mathbb{R}^{m} \mid \mu^{T} g_{x}^{i, j}=0, i \in I\left(x^{j}\right)\right\}$ ist die quadratische Form $\mu^{T}\left(e_{x x}^{j}-\sum_{i \in I\left(x^{\prime}\right)} w_{i}^{i} g_{x x}^{i, j}\right) \mu$ negativ definit.

Hilfssatz 1. Zu jedem $x^{j} \in E$ gibt es eine Umgebung $U_{j}$, so da $e\left(p^{0}, x\right)<e\left(p^{0}, x^{j}\right)$ für jedes $x \in U_{j} \cap B, x \neq x^{j}$.

Einen Beweis findet man etwa in [1] oder [2], wo gezeigt wird, daß (II) hinreichend dafür ist, $\mathrm{da} B e\left(p^{0}, x\right)$ in jedem Punkt von $E$ ein starkes relatives Maximum besitzt.

Aus Hilfssatz 1 und der Tatsache, da $B$ kompakt ist, folgt unmittelbar

Hilfssatz 2. $E$ enthält nur endlich viele Punkte: $E=\left\{x^{1}, \ldots, x^{r}\right\}, r<\infty$.

\section{Zwei Sätze über Ungleichungen}

Es sei $M$ eine reelle $m \times n$-Matrix, $b \in \mathbb{R}^{m}$ ein Vektor. Uber die Lösbarkeit des Systems von Ungleichungen $M x<b$ gelten folgende Sätze:

Hilfssatz 3. (Transpositionssatz von Gordan, vgl. etwa [4].): Das homogene System $M x<0$ ist genau dann unlösbar, wenn es einen Vektor $u \geqq 0, u \neq 0, u \in \mathbb{R}^{m}$, gibt mit $M^{T} u=0$.

Hilfssatz 4. Das inhomogene System ist genau dann unlösbar, wenn es einen Vektor $u \geqq 0, u \neq 0, u \in \mathbb{R}^{m}$, gibt mit $M^{T} u=0, u^{T} b \leqq 0$.

Hilfssatz 4 ist eine Spezialisierung des Satzes von Kuhn-Fourier ([4]). 


\section{Die Maxima der Fehlerfunktion}

Sei $p^{0} \in P$ ein fester Punkt. $Z u$ beliebigen Vektoren $\xi, \eta \in \mathbb{R}^{n}$ gibt es dann ein $t^{*}>0$, so daß $p(t)=p^{0}+t \xi+\frac{t^{2}}{2} \eta, t \in\left[-t^{*}, t^{*}\right]$ ein ganz in $P$ verlaufender Weg ist. Im folgenden sei stets $t \in\left[-t^{*}, t^{*}\right]$, ohne daß wir das jedesmal ausdrücklich sagen.

Für $t=0$ nimmt $e(p(t), x)$ genau in den Punkten $x^{1}, \ldots, x^{r}$ von $E$ seinen maximalen Wert an. Es ist zu erwarten, da $e(p(t), x)$ für kleine $t$ lokale Maxima in Umgebungen der Punkte $x^{i}$ besitzt. Um dies genauer zu untersuchen, definieren wir für ein nunmehr festes $x^{j} \in E$ die vektorwertige Funktion

$$
\Phi^{j}(x, w ; t)=\left(\begin{array}{c}
e_{x}(p(t), x)-\sum_{i=1}^{m_{j}} w_{i} g_{x}^{i}(x) \\
-g^{1}(x) \\
\cdots \\
-g^{m_{j}}(x)
\end{array}\right)\left(m+m_{j} \text { Komponenten }\right)
$$

wobei wir o.B.d.A. $I\left(x^{j}\right)=\left\{1, \ldots, m_{j}\right\}, m_{j} \leqq m$, angenommen haben. Die $w_{i}$ sind $z$ u dem Vektor $w=\left(w_{1}, \ldots, w_{m}\right)^{T}$ zusammengefaßt. Für $t=0, x=x^{j}$ und $w=w^{j}=\left(w_{1}^{i}, \ldots, w_{m}^{j}\right)^{T}$ gilt nach (II)

$$
\Phi^{j}\left(x^{j}, w^{j} ; 0\right)=0 .
$$

Die Jakobi-Matrix bez. $x$ und $w$ im Punkt $x=x^{j}, w=w^{j}, t=0$ wird

$$
\boldsymbol{\Phi}_{(x, w)}^{j}\left(x^{i}, w^{i} ; 0\right)=\left(\begin{array}{c|ccc}
e_{x x}^{j}-\sum_{i=1}^{m} w_{i}^{i} g_{x x}^{i, j} & -g_{x}^{1, j} \ldots & -g_{x}^{m, j} \\
\hline\left(-g_{x}^{1, j}\right)^{T} & 0 & \ldots & 0 \\
\ldots & & \ldots & \\
\left(-g_{x}^{m, j}\right)^{T} & 0 & \ldots & 0
\end{array}\right)=\left(\begin{array}{c|c}
M_{i} & G_{i}^{T} \\
\hline G_{j} & 0
\end{array}\right) .
$$

Nach (II) ist $M_{j}$ negativ definit auf dem Teilraum $T_{j}=\left\{\boldsymbol{\mu} \mid G_{j} \mu=0\right\}$.

Nach (I) hat $G_{j}$ den Rang $m_{\eta}$, so daß aus $G_{j}^{T} \alpha=0, \alpha \in \mathbb{R}^{m}, \alpha=0$ folgt.

Betrachte das homogene Gleichungssystem $\left(\begin{array}{cc}M_{j} & G_{i}^{T} \\ G_{j} & 0\end{array}\right)\left(\begin{array}{l}\mu \\ \alpha\end{array}\right)=0$.

Angenommen, $\left(\begin{array}{l}\mu_{0} \\ \alpha_{0}\end{array}\right)$ sei eine nichttriviale Lösung. $\mu_{0}=0$ ist nicht möglich, da sonst $G_{j}^{T} \alpha_{0}=0, \alpha_{0} \neq 0$, geltén müßte. Wegen $G_{j} \mu_{0}=0$ ist $\mu_{0}^{T} M_{j} \mu_{0}<0$. Es würde folgen

$$
0=\mu_{0}^{T}\left(M_{j} \mu_{0}+G_{j}^{T} \alpha_{0}\right)=\mu_{0}^{T} M_{i} \mu_{0}<0,
$$

was unmöglich ist. Somit hat obiges System nur die triviale Lösung und daher ist die Matrix $\Phi_{(x, w)}^{j}\left(x^{j}, w^{j} ; 0\right)$ nichtsingulär.

Nach einem bekannten Satz tiber implizite Funktionen gilt deshalb: Es gibt Umgebungen $U_{x t}, U_{w i}$ und $U_{t}$ von $x^{i}$, $w^{i}$ und $t=0$ und zweimal stetig differenzierbare $x^{j}(t), w^{j}(t)$, definiert in $U_{t}$ und mit Werten in $U_{z j}, U_{w t}$, so daß für jedes $t \in U_{i}$ gilt:

Genau dann ist $\Phi^{j}(x, w ; t)=0$ für $x \in U_{x^{\prime}}, w \in U_{w i}$, wenn $x=x^{j}(t), w=w^{j}(t)$. 
Ferner gilt

$$
\left(\begin{array}{c}
\dot{x}^{j}(0) \\
\dot{w}^{j}(0)
\end{array}\right)=-\left(\begin{array}{cc}
M_{j} & G_{j}^{T} \\
G_{j} & 0
\end{array}\right)^{-1} \cdot \frac{\partial}{\partial t} \Phi^{j}\left(x^{j}, w^{j} ; 0\right) .
$$

Daher kann $\mu_{j}=\dot{x}^{i}(0)$ eindeutig aus dem folgenden Gleichungssystem berechnet werden

$$
\left(\begin{array}{cc}
M_{j} & G_{i}^{T} \\
G_{i} & 0
\end{array}\right)\left(\begin{array}{c}
\mu_{j} \\
\dot{w}^{j}(0)
\end{array}\right)=\left(\begin{array}{c}
-e_{x p}^{i} \xi \\
0
\end{array}\right)
$$

Ersichtlich ist $\left(g_{x}^{i, j}\right)^{T} \mu_{j}=0, i=1, \ldots, m_{j}$. Weiter folgt für $i=1, \ldots, m_{j}$ : $0=g^{i}\left(x^{j}(t)\right)=\underbrace{g^{i, j}}_{=0}+\underbrace{t \mu_{j}^{T} g_{x}^{i, j}}_{=0}+\frac{t^{2}}{2}\left[\lambda_{j}^{T} g_{x}^{i, j}+\mu_{j}^{T} g_{x x}^{i, j} \mu_{j}\right]+o\left(t^{2}\right)$, mit $\lambda_{j}=\ddot{x}^{j}(0)$. Grenzübergang $t \rightarrow 0$ zeigt, daß $\lambda_{j}$ dem folgenden linearen Gleichungssystem genügt

$$
\lambda_{j}^{T} g_{x}^{i, j}=-\mu_{j}^{T} g_{x x}^{i, j} \mu_{j}, \quad i=1, \ldots, m_{j} .
$$

Da die $g_{x}^{i, j}, i=1, \ldots, m_{j}$, linear unabhängig sind, ist (2) stets lösbar.

Man betrachte nun die quadratische Form

$$
q_{j}(\mu ; t)=\mu^{T}\left[e_{x x}\left(p(t), x^{j}(t)\right)-\sum_{i=1}^{m_{y}} w_{i}^{j}(t) g_{x x}^{i}\left(x^{j}(t)\right)\right] \mu
$$

auf dem Teilraum $T_{j}(t)=\left\{\mu \mid \mu^{T} g_{x}^{i}\left(x^{j}(t)\right)=0, i=1, \ldots, m_{j}\right\}$.

Nach (II) ist $q_{j}(\mu ; 0)$ auf $T_{j}(0)$ negativ definit. Da sowohl $q_{j}$ als $T_{j}$ stetig von $t$ abhängen, gibt es ein $t_{j}^{*}>0$, so daß $q_{j}(\mu ; t)$ auf $T_{j}(t)$ negativ definit ist für $t \in\left[0, t_{j}^{*}\right]$. Sei nun $t_{j}=\min \left\{t_{j}^{*}, \max \left\{t \mid[0, t] \leq U_{t}\right\}\right\}$ und $t_{0}^{\prime}=\min _{j=1, \ldots, r}\left\{t_{j}\right\}$. Dann gilt (vgl. Hilfssatz 1): Maxima.

Für jedes $t \in\left[0, t_{0}^{\prime}\right]$ hat $e(p(t), x)$ in $B \cap \bigcup_{j=1}^{r} U_{x j}$ genau in $x^{1}(t), \ldots, x^{r}(t)$ lokale
xima.

Da weiter mit einem gewissen $d>0$ für $x \in B \sim \bigcup_{i=1}^{\prime} U_{x^{\prime}}$

$$
e\left(p^{0}, x\right) \leqq\left\|e\left(p^{0}, \cdot\right)\right\|-d
$$
gilt, gibt es ein $t_{0}^{\prime \prime}$, so daß $e(p(t), x)<\left\|e\left(p^{0}, \cdot\right)\right\|$ für $t \in\left[0, t_{0}^{\prime \prime}\right]$ und $x \in B \sim \bigcup_{j=1}^{\prime} U_{x \neq}$.
Damit haben wir folgenden Satz bewiesen:

Satz 1. Sei $p(t)=p^{0}+t \xi+\frac{t^{2}}{2} \eta, t_{0}=\min \left\{t_{0}^{\prime}, t_{0}^{\prime \prime}\right\}$. Dann hat für $t \in\left[0, t_{0}\right]$ $e(p(t), x)$ in $\bigcup_{j=1}^{\prime} U_{x^{\prime}}$ genau die lokalen Maxima $x^{1}(t), \ldots, x^{\gamma}(t)$ und in $B \sim \bigcup_{j=1}^{j} U_{x^{\prime}}$ gilt $e(p(t), x)<\left\|e\left(p^{0}, \cdot\right)\right\|$. Hierbei ist

$$
x^{j}(t)=x^{j}+t \mu_{i}+\frac{t^{2}}{2} \lambda_{i}+o\left(t^{2}\right)
$$

wo $\mu_{j}$ durch (1) eindeutig bestimmt ist und $\lambda_{j}$ (2) genügt.

Um das Verhalten der Fehlerfunktion in den lokalen Maxima zu untersuchen, entwickeln wir diese nach Potenzen von $t$ :

$$
e\left(p(t), x^{i}(t)\right)=e^{j}+t e^{j}+\frac{t^{2}}{2} \ddot{e}^{i}+o\left(t^{2}\right)
$$


Es gilt $e^{i}=\left\|e\left(p^{0}, \cdot\right)\right\|$,

und

$$
\dot{e}^{j}=\xi^{T} e_{p}^{j}+\mu_{j}^{T} e_{x}^{j}=\xi^{T} e_{p}^{j}+\sum_{i=1}^{m} w_{i}^{j} \underbrace{\mu_{j}^{T} g_{x}^{i, j}}_{=0}=\xi^{T} e_{p}^{j}
$$

$$
\ddot{e}^{j}=\eta^{T} e_{p}^{j}+2 \xi^{T} e_{p x}^{j} \mu_{j}+\xi^{T} e_{p p}^{j} \xi+\lambda_{j}^{T} e_{x}^{j}+\mu_{j}^{T} e_{x x}^{j} \mu_{j} .
$$

Mit $e_{x}^{i}=\sum_{i=1}^{m g} w_{i}^{j} g_{x}^{i, j}$ und (2) folgt

$$
e^{-j}=\eta^{T} e_{p}^{j}+2 \xi^{T} e_{p x}^{j} \mu_{j}+\xi^{T} e_{p p}^{j} \xi+\mu_{j}^{T} M_{j} \mu_{j} .
$$
Multipliziert man (1) von links mit $\left(\begin{array}{c}\mu_{j} \\ \dot{\omega}^{j}(0)\end{array}\right)^{T}$, so folgt $\mu_{j}^{T} M_{j} \mu_{j}=-\mu_{j}^{T} e_{x p}^{j} \xi$, so da $B$
wir endlich erhalten

Somit ergibt sich

$$
\ddot{e}^{j}=\eta^{T} e_{p}^{j}+\xi^{T} e_{p p}^{j} \xi-\mu_{j}^{T} M_{j} \mu_{j}
$$

$$
e\left(p(t), x^{i}(t)\right)=\left\|e\left(p^{0}, \cdot\right)\right\|+t \xi^{T} e_{p}^{j}+\frac{t^{2}}{2}\left[\eta^{T} e_{p}^{j}+\xi^{T} e_{p p}^{j} \xi-\mu_{j}^{T} M_{j} \mu_{j}\right]+o\left(t^{2}\right)
$$

mit $M_{i}=\left(e_{x x}^{j}-\sum_{i \in I\left(x^{j}\right)} w_{i}^{j} g_{x x}^{i, j}\right)$ und $\mu_{j}$ aus (1). (Die Indexmenge $I\left(x^{j}\right)$ ist hier wieder allgemein genommen.)

\section{Notwendiges Kriterium}

Wir nehmen an, $p^{0}$ sei eine lokal beste Approximation. Nach den Betrachtungen des vorigen Abschnitts muß dann für jedes $p(t)=p^{0}+t \xi+\frac{t^{2}}{2} \eta$ gelten: Es gibt kein $t_{1}>0$, so daß

$$
e\left(p(t), x^{j}(t)\right)<\left\|e\left(p^{0}, \cdot\right)\right\| \quad \text { für } j=1, \ldots, r, t \in\left[0, t_{1}\right] .
$$

Mit (3) folgt hieraus unmittelbar, daß das System $\xi^{T} e_{p}^{j}<0, j=1, \ldots, r$, keine Lösung $\xi$ hat. Nach Hilfssatz 3 ist dies genau dann der Fall, wenn es Zahlen $u_{j} \geqq 0, j=1, \ldots, r$, nicht alle $u_{j}=0$, gibt, so daß

$$
\sum_{j=1}^{\infty} u_{j} e_{p}^{i}=0
$$

Sei $\xi$ eine Lösung von $\xi^{T} e_{p}^{j} \leqq 0$ mit $\xi^{T} e_{p}^{j}=0$ genau für $j=1, \ldots, r_{1}$. Dann ist $u_{j}=0, j=r_{1}+1, \ldots, r$, da andernfalls folgen würde

$$
0=\xi^{T}\left(\sum_{j=1}^{r} u_{j} e_{p}^{j}\right)=\sum_{j=r_{1}+1}^{r} u_{j} \xi^{T} e_{p}^{j}<0 .
$$

Wegen $e\left(p(t), x^{j}(t)\right)<\left\|e\left(p^{0}, \cdot\right)\right\|, j=r_{1}+1, \ldots, r$, für hinreichend kleine $t$, darf es kein $t_{1}>0$ geben, so daB für $j=1, \ldots, y_{1}$

$$
e\left(p(t), x^{i}(t)\right)-\left\|e\left(p^{0}, \cdot\right)\right\|=\frac{t^{2}}{2}\left[\eta^{T} e_{p}^{j}+\xi^{T} e_{p p}^{j} \xi-\mu_{j}^{T} M_{i} \mu_{j}\right]+o\left(t^{2}\right)<0
$$


für $t \in\left[0, t_{1}\right]$. Hieraus folgt, daß das System

$$
\eta^{T} e_{p}^{j}<-\xi^{T} e_{p p}^{j} \xi+\mu_{j}^{T} M_{j} \mu_{j}, \quad j=1, \ldots, r_{1}
$$

keine Lösung $\eta$ haben darf (man beachte, daß die aus (1) bestimmten $\mu_{j}$ nicht von $\eta$, sondern nur von $\xi$ abhängen, und zwar linear: $\mu_{j}=S_{j} \xi$ mit gewissen $m \times n$ Matrizen $S_{j}$ ).

Anwendung von Hilfssatz 4 liefert nun unmittelbar

Satz 2. $p^{0}$ sei eine lokal beste Approximation. Dann gibt es zu jedem $\xi$ mit $\xi^{T} e_{p}^{j} \leqq 0, j=1, \ldots, r$, Zahlen $u_{j} \geqq 0$ (nicht alle gleich Null), so da

und

$$
\sum_{i=1}^{r} u_{j} e_{p}^{j}=0
$$

$$
q(\xi)=\xi^{T}\left\{\sum_{j=1}^{r} u_{j} e_{p p}^{j}\right\} \xi-\sum_{j=1}^{r} u_{j} \mu_{j}^{T} M_{j} \mu_{j} \geqq 0,
$$

wo die $\mu_{j}$ durch (1) bestimmt sind und mit $M_{j}=e_{x x}^{j}-\sum_{i \in I(x \prime)} w_{i}^{j} g_{x x}^{i, j}$.

\section{Es gilt}

\section{Hinreichendes Kriterium}

Satz 3. Wenn es zu jedem $\xi$ mit $\xi^{T} e_{p}^{j} \leqq 0, j=1, \ldots, r$, Zahlen $u_{j} \geqq 0$, nicht alle gleich Null, gibt, so daß

$$
\sum_{j=1}^{\infty} u_{j} e_{p}^{i}=0
$$

und $q(\xi)>0$, falls $\xi \neq 0$, dann ist $p^{0}$ eine lokal beste Approximation.

Beweis. Es genügt, zu zeigen, daß es zu jedem $\xi \in \mathbb{R}^{n}$ ein $j \in\{1, \ldots, r\}$ gibt mit $e\left(p(t), x^{j}(t)\right)>\left\|e\left(p^{0}, \cdot\right)\right\|$ für $t$ in einem Intervall $\left[0, t_{0}\right]$. Hierbei ist $p(t)=p^{0}$ $+t \xi+\frac{t^{2}}{2} \eta$ mit einem beliebigen $\eta \in \mathbb{R}^{n}$. Ist $\xi^{T} e_{p}^{j_{j}}>0$ für ein $j_{0} \in\{1, \ldots, r\}$, so ist dies nach (3) sicher richtig. Sei also $\xi^{T} e_{p}^{j} \leqq 0, j=1, \ldots, r$. Aus den Voraussetzungen des Satzes folgt, daß das System $\xi^{T} e_{p}^{j}<0, j=1, \ldots, r$, nicht lösbar ist (Hilfssatz 3).

Es gibt somit stets Indices $j$ mit $\xi^{T} e_{p}^{j}=0$, o.B.d.A. $\xi^{T} e_{p}^{j}=0$ für $j=1, \ldots, r_{1}$. Nach Voraussetzung gibt es $u_{j} \geqq 0$, so $\mathrm{da} \beta \sum_{j=1}^{r} u_{j} e_{p}^{j}=0$ und

$$
\sum_{j=1}^{t} u_{j}\left[\xi^{T} e_{p p}^{j} \xi-\mu_{j}^{r} M_{j} \mu_{j}\right]>0
$$

wobei, wie im letzten Abschnitt gezeigt wurde, $u_{j}=0, j=r_{1}+1, \ldots, r$.

Also gibt es mindestens ein $k \in\left\{1, \ldots, r_{1}\right\}$ mit $\xi^{T} e_{p p}^{k} \xi-\mu_{k}^{T} M_{k} \mu_{k}>0$. Nach (3) gilt somit

$$
e\left(p(t), x^{k}(t)\right)=\left\|e\left(p^{0}, \cdot\right)\right\|+\frac{t^{2}}{2}\left[\xi^{T} e_{p p}^{k} \xi-\mu_{k}^{T} M_{k} \mu_{k}\right]+o\left(t^{2}\right)>\left\|e\left(p^{0}, \cdot\right)\right\|
$$

für hinreichend kleine $t$. Damit ist Satz 3 bewiesen. 


\section{Ein Beispiel}

Wir betrachten das Problem, die Funktion $f(x)=1-x^{2}$ durch eine Funktion $a(p, x)=\frac{1}{2} p^{2}-2 p x, p \in \mathbb{R}=P$, im Intervall $[-1,1] \mathrm{zu}$ approximieren. Es ist $e(p, x)=\left|1-x^{2}-\frac{1}{2} p^{2}+2 p x\right|$.

Wir zeigen mit Hilfe von Satz 3 , daß $p^{0}=0$ eine lokal beste Approximation ist: die Menge $E$ besteht hier nur aus dem Punkt $x^{1}=0$. Man berechnet

$$
q(\xi)=u \xi^{2}\left(e_{p p}^{1}-\left(\frac{e_{p x}^{1}}{e_{x x}^{1}}\right)^{2} e_{x x}^{1}\right)=u \xi^{2}\left(-1-\left(\frac{2}{2}\right)^{2}(-2)\right)=u \xi^{2}>0
$$

für alle $\xi \neq 0, u>0$. Ferner ist $e_{p}^{1}=0$, also $u e_{p}^{1}=0$ für alle $u \geqq 0$. Nach Satz 3 ist $p^{0}=0$ somit eine lokal beste Approximation. Es ist leicht zu beweisen, daß $p^{0}=0$ sogar die global beste Approximation ist.

Bemerkenswert an diesem Beispiel ist, daß $p^{0}=0$ keine lokal beste Approximation ist, wenn wir statt auf $[-1,1]$ auf irgendeiner diskreten Teilmenge $\left\{y^{1}, \ldots, y^{\prime}\right\}$ von $[-1,1]$, die den Punkt $x=0$ enthält, approximieren. Dies ist einfach zu sehen, wenn man beachtet, daß die Funktion $e(p, 0)$ in $p=0$ ein Maximum besitzt.

Trotz dieses Sachverhalts konnten wir an Hand von Satz 3 durch alleinige Betrachtung des Punktes $p^{0}=0, x=0$, auf eine lokal beste Approximation schließen.

Das Kriterium von Kolmogoroff versagt bei diesem Beispiel: es müßte für jedes $p \in \mathbb{R}$ gelten (vgl. [3], Satz 81):

$$
[f(0)-a(0,0)][a(p, 0)-a(0,0)] \leqq 0 .
$$

Es ist jedoch $[f(0)-a(0,0)][a(p, 0)-a(0,0)]=\frac{1}{2} p^{2}>0$ für jedes $p \neq 0$.

\section{Schlußbemerkungen}

Wir können nun etwas mehr sagen zu dem im Abschnitt 3 angeschnittenen Problem, wie man die Voraussetzung (I) abschwächen könnte. Unmittelbar deutlich ist, daß es ausreicht, die lineare Unabhängigkeit der $g_{x}^{i}(x), i \in I(x)$, nur für $x \in E \mathrm{zu}$ fordern. Hiervon wurde Gebrauch gemacht, um die Wege $x^{j}(t)$ so zu bestimmen, daß $g\left(x^{j}(t)\right) \equiv 0$ gilt für $j \in I\left(x^{j}\right)$. Bei linear abhängigen $g_{x}^{i, j}$ ist dies i.all. nicht mehr möglich. Man muß dann auf andere Weise versuchen, die Wege, längs denen die Extrema der Fehlerfunktion abwandern, zu beschreiben. Im wesentlichen dasselbe Problem erhält man beim Versuch, die Forderung (II) abzuschwächen.

Weiter ist zu bemerken, daß diskrete Bereiche $B$ nicht so beschrieben werden können, daß (I) erfüllt ist. Es ist jedoch sehr einfach, für diskrete Approximationsprobleme entsprechende Kriterien aufzustellen: die Punkte, in denen $e\left(p^{0}, x\right)$ maximal ist, bleiben bei hinreichend kleinen Veränderungen von $p$ die einzigen Kandidaten für Extrema von $e(p, x)$. Man erhält genau die Sätze 2 und 3, wobei nur alle $\mu_{i}$ gleich Null zu nehmen sind (vgl. [1]).

Eine Verallgemeinerung von praktischer Bedeutung wäre die folgende: statt eines offenen Parameterbereiches $P$ könnte man Bereiche der Form

$$
P:=Q \cap\left\{p \in \mathbb{R}^{n} \mid h(p, y) \leqq 0, y \in Y\right\}
$$


betrachten, wobei $Q \leq \mathbb{R}^{*}$ eine offene und $Y$ eine kompakte Teilmenge eines $\mathbb{R}^{\prime}, r \geqq 1$, ist. Hierdurch wäre es möglich, die Theorie auf allgemeinere Aufgabentypen wie etwa die einseitige oder die monotone Approximation auszudehnen.

\section{Literatur}

1. Hettich, R.: Extremalkriterien für Optimierungs- und Approximationsaufgaben. Technische Hogeschool Twente, Enschede, Dissertation, 1973

2. MacCormick, G. P.: Second order conditions for constrained minima. SIAM J. Appl. Math. 15, 641-652 (1967)

3. Meinardus, G.: Approximation von Funktionen und ihre numerische Behandlung. Berlin-Heidelberg-New York: Springer 1964

4. Stoer, J., Witzgall, Ch.: Convexity and optimization in finite dimensions $\mathbf{I}$. Berlin-Heidelberg-New York: Springer 1970

5. Wetterling, W.: Definitheitsbedingungen für relative Extrema bei Optimierungsund Approximationsaufgaben. Num. Math. 15, 122-136 (1970)

R. Hettich

Technische Hogeschool Twente

Onderafdeling der Toegepaste Wiskunde

Enschede, Pustbus 217

Niederlande 